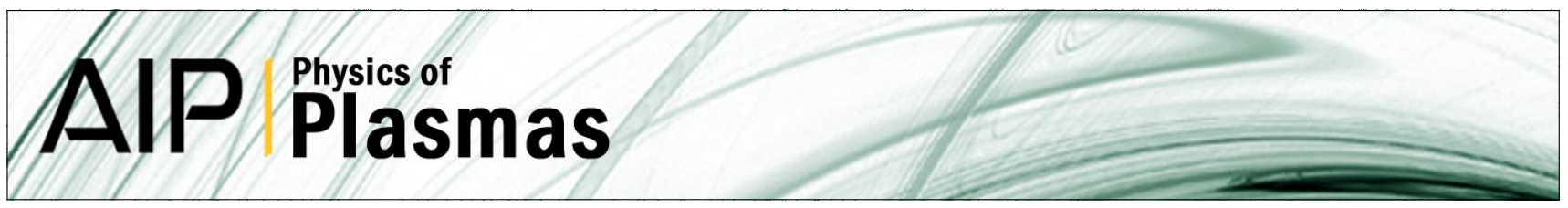

\title{
Suprathermal ion transport in simple magnetized torus configurations
}

K. Gustafson, P. Ricci, A. Bovet, I. Furno, and A. Fasoli

Citation: Phys. Plasmas 19, 062306 (2012); doi: 10.1063/1.4725420

View online: http://dx.doi.org/10.1063/1.4725420

View Table of Contents: http://pop.aip.org/resource/1/PHPAEN/v19/i6

Published by the American Institute of Physics.

\section{Related Articles}

Application of a three-dimensional model for a study of the energy transfer of a high-pressure mercury horizontal lamp

Phys. Plasmas 19, 063504 (2012)

Hyper-resistivity and electron thermal conductivity due to destroyed magnetic surfaces in axisymmetric plasma equilibria

Phys. Plasmas 19, 062502 (2012)

Quasilinear transport modelling at low magnetic shear

Phys. Plasmas 19, 062305 (2012)

Parallel transport of long mean-free-path plasma along open magnetic field lines: Parallel heat flux

Phys. Plasmas 19, 062501 (2012)

A coarse-grained kinetic equation for neutral particles in turbulent fusion plasmas

Phys. Plasmas 19, 060701 (2012)

\section{Additional information on Phys. Plasmas}

Journal Homepage: http://pop.aip.org/

Journal Information: http://pop.aip.org/about/about_the_journal

Top downloads: http://pop.aip.org/features/most_downloaded

Information for Authors: http://pop.aip.org/authors

\section{ADVERTISEMENT}

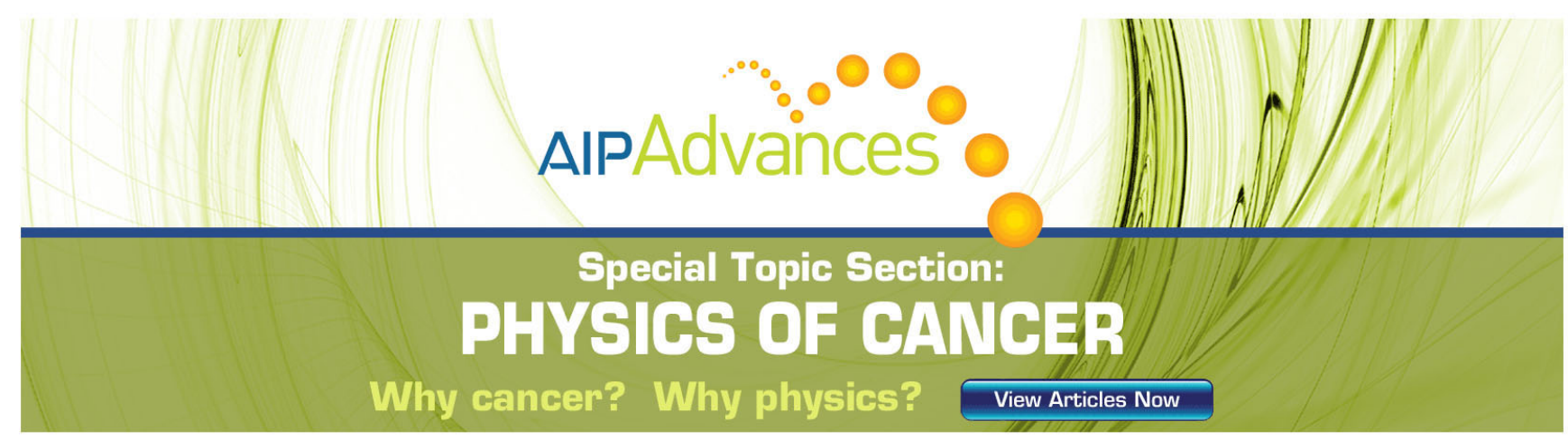




\title{
Suprathermal ion transport in simple magnetized torus configurations
}

\author{
K. Gustafson, ${ }^{\text {a) }}$ P. Ricci, A. Bovet, I. Furno, and A. Fasoli \\ Ecole Polytechnique Fédérale de Lausanne (EPFL), Centre de Recherches en Physique des Plasmas, \\ Association Euratom-Confédération Suisse, CH-1015 Lausanne, Switzerland
}

(Received 20 December 2011; accepted 24 April 2012; published online 14 June 2012)

\begin{abstract}
Inspired by suprathermal ion experiments in the basic plasma experiment TORPEX, the transport of suprathermal ions in ideal interchange mode turbulence is theoretically examined in the simple magnetized torus configuration. We follow ion tracer trajectories as specified by ideal interchange mode turbulence imported from a numerical simulation of drift-reduced Braginskii equations. Using the variance of displacements, $\sigma^{2}(t) \sim t^{\gamma}$, we find that $\gamma$ depends strongly on suprathermal ion injection energy and the relative magnitude of turbulent fluctuations. The value of $\gamma$ also changes significantly as a function of time after injection, through three distinguishable phases: ballistic, interaction, and asymmetric. During the interaction phase, we find the remarkable presence of three regimes of dispersion: superdiffusive, diffusive, and subdiffusive, depending on the energy of the suprathermal ions and the amplitude of the turbulent fluctuations. We contrast these results with those from a "slab" magnetic geometry in which subdiffusion does not occur during the interaction phase. Initial results from TORPEX are consistent with data from a new synthetic diagnostic used to interpret our simulation results. The simplicity of the simple magnetized torus makes the present work of interest to analyses of more complicated contexts ranging from fusion devices to astrophysics and space plasma physics. [http://dx.doi.org/10.1063/1.4725420]
\end{abstract}

\section{INTRODUCTION}

We present a study of suprathermal ion dynamics in the simple magnetized torus (SMT) configuration, ${ }^{1-3}$ in which a vertical magnetic field, $B_{v}$, superimposed on a toroidal magnetic field, $B_{\phi}$, creates helicoidal field lines terminating on the vessel. In this configuration, turbulence driven by magnetic curvature and plasma gradients causes the plasma to diffuse radially, while it is lost to the vessel through parallel flows. The SMT experimental setup, in which ions are subject to a non-uniform, curved magnetic field and plasma turbulence, is an ideal testbed for the study of the interplay of several phenomena affecting suprathermal ion dynamics. Parameter scans are easier and nonlinear dynamical behavior can be diagnosed in greater detail when compared to fusion-prototype devices. Moreover, SMT plasmas are well-understood after being subjected to linear instability analysis ${ }^{4}$ and global turbulence simulations, ${ }^{5}$ making a thorough experimental study possible in tandem with analytical progress.

Our work is inspired by fast ion experiments in TORPEX, ${ }^{3}$ an SMT used for understanding basic plasma turbulence phenomena. Recently, the TORPEX team has been conducting suprathermal ion experiments ${ }^{6}$ with an emitter of energetic lithium ions. ${ }^{7}$ The ion energy and beam orientation are tunable. The current from the emitted ion beam is detected by a double-gridded energy analyzer, which can be moved in the plane perpendicular to the magnetic field to measure a spatially resolved profile of the suprathermal ion current density. This current density profile gives information about the spreading of suprathermal ions due to the forces applied by steady-state magnetic fields and fluctuating electric fields.

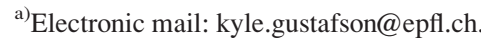

Expanding on results presented in Ref. 8, the goal of the present paper is to construct a theoretical framework for understanding the behavior of suprathermal ions in the SMT, depending on ion energy and turbulence fluctuation level. We also present an initial comparison with experimental data from TORPEX. Due to its relative simplicity, the SMT successfully disentangles factors that determine suprathermal ion dispersion. Beyond interpretation of TORPEX measurements, this new framework in the generality of the SMT configuration is therefore useful to analyses of more complicated configurations, ranging from fusion devices ${ }^{9,10}$ to astrophysics $^{11,12}$ and space plasma physics. ${ }^{13}$

Our study is based on numerical integration of the charged-particle equation of motion in the SMT environment for a range of injection energies and turbulence fluctuation amplitudes. We consider suprathermal ions as tracer particles, such that they do not influence background fields. This is a reasonable approximation in TORPEX because the density of suprathermal ions is very small compared with the plasma density. The tracer approach allows more efficient computations by avoiding the recalculation of Maxwell's equations as the tracers propagate. We use SMT turbulence simulations reported recently ${ }^{14,15}$ to provide the timedependent electric fields required to integrate realistic trajectories with the full Lorentz equation of motion. Our primary diagnostic tool is the time-dependent variance of suprathermal ion displacements, $\sigma^{2}(t) \sim t^{\gamma}$, with which we measure the dispersion of tracer ions.

We focus on fast ion spatial spreading in the direction of the major radius, $\mathbf{e}_{R}$. We find that a nondiffusive model, defined by $\gamma \neq 1$, is necessary to describe the dispersion of ions in our study. Our simulations show that, in fact, suprathermal ion dispersion in the SMT begins with a brief 
ballistic phase, for which $\gamma_{R} \simeq 2$. Then, a turbulence interaction phase occurs, with the value of $\gamma_{R}$ depending nontrivially on beam energy and turbulence fluctuation amplitude. In some cases, a third phase is observed for which $\gamma_{R}$ increases from $\gamma_{R}<1$ to $\gamma_{R} \sim 1$ but with significant asymmetry in the radial mean position $(d\langle\delta R\rangle / d t<0)$ of the ion beam. We provide analytical estimates for the duration of the ballistic phase and the value of $\gamma_{R}$ in the interaction phase, as well as details about the asymmetric phase.

While the main focus of the present work is the SMT configuration, we also consider a "slab" configuration to reach a better understanding of the dominant influences on suprathermal ion transport. In this context, a "slab" configuration features a linear, homogeneous magnetic field, which can be viewed as an SMT in the limit of $R_{0} \rightarrow \infty$, where $R_{0}$ is the SMT major radius. A slab field allows separation of curvature and $\nabla B$ from finite Larmor radius effects. We note that a suprathermal ion diagnostic similar to the one in TORPEX is used in the slab-like LAPD device. ${ }^{16}$ We therefore expect our results to provide insights for LAPD as well.

Previous studies of tracer particle dispersion in plasmas have examined nondiffusive dispersion in stochastic fields, typically with an $\mathbf{E} \times \mathbf{B}$ approximation for the particle velocity ${ }^{17}$ and sometimes with finite Larmor radius effects. ${ }^{18}$ These approximations to the full Lorentz motion are typically effective for small Larmor radius and large separation between the cyclotron frequency and turbulence fluctuation frequencies, and they allow a calculation that covers large temporal scales. This is necessary, for example, to describe the neutral beam slowing down time in tokamaks (see, e.g., Ref. 19). For our study, we use the full Lorentz force, which correctly describes the effects of turbulence on the suprathermal ions across scales, including those comparable to $\rho$ and $\Omega_{i}$.

This paper continues with a review of the properties of SMT plasma turbulence in Sec. II. In Sec. III, we consider the main elements characterizing suprathermal ion motion in the SMT configuration. We take a detailed tour of test particle dispersion results at short, medium, and long times as a function of beam injection energy and turbulence amplitude in Sec. IV. The first comparison of suprathermal ion theoretical and experimental results in TORPEX is given in Sec. V, which includes the use of a new synthetic diagnostic for suprathermal ion current density measurement. Finally, conclusions are given in Sec. VI.

\section{PLASMA DYNAMICS IN THE SIMPLE MAGNETIZED TORUS CONFIGURATION}

Plasma behavior in the SMT results from the interplay of plasma sources, losses at the vessel wall, and turbulence. In TORPEX, a localized source of plasma on the high-field side of the torus is created directly with electron-cyclotron and upper-hybrid microwave resonances. Turbulence, driven by magnetic field curvature and plasma gradients, causes bulk plasma transport perpendicular to B. Measurements show that TORPEX has large amplitude turbulent fluctuations, with $\delta n / n \lesssim 1$. The dominant modes have $k_{\perp} \rho_{s} \lesssim 1$ and $k_{\|} \ll k_{\perp}$, with frequencies $\omega \ll \Omega_{c i}$.
In the experimentally relevant limits of $B_{v} \ll B_{\phi}$, $\beta \ll 1$, and $T_{i} \ll T_{e}$, the theoretical study of turbulence in the SMT has recently resulted in significant advances using the drift-reduced Braginskii two-fluid equations. ${ }^{20}$ Simulations of these equations have been carried out by evolving the plasma dynamics, which results from the interaction between heat and particle sources, losses at the vessel, and turbulence, without separating equilibrium and fluctuating quantities. ${ }^{5}$ These simulations have been subjected to extensive validation against probe data from TORPEX. ${ }^{21,22}$

A number of modes are present in the SMT including the ideal interchange, resistive interchange, and drift-wave modes. In the present discussion, we focus on the ideal interchange regime, which is dominant for sufficiently low values of plasma resistivity and sufficiently high values of $B_{v}{ }^{5}$ Ideal interchange turbulence is characterized by $k_{\|}=0$. Therefore, the vertical wavelength is constrained by the return of the field line on the poloidal plane to be a multiple of $k_{\Delta}=2 \pi / \Delta$. Here, we define $\Delta=2 \pi R_{0} B_{v} / B_{\phi}$ as the return distance of the field line in the poloidal plane.

Ideal-interchange turbulence can be described through global two-dimensional simulations in the plane perpendicular to the magnetic field. It is therefore practical to consider a field-line following coordinate system $\left(\mathbf{e}_{R}, \mathbf{e}_{\|}, \mathbf{e}_{z}\right)$, where the unit vectors $\mathbf{e}_{R}, \mathbf{e}_{\|}$, and $\mathbf{e}_{z}$ are in the radial, parallel to $\mathbf{B}$, and perpendicular to both $\mathbf{e}_{R}$ and $\mathbf{e}_{\|}$directions, respectively. We note that $\mathbf{e}_{\|}$is tilted relative to the lab-frame horizontal by an angle $\theta \equiv \tan ^{-1}\left(B_{v} / B_{\phi}\right) \ll 1$. Therefore, $\mathbf{e}_{z}$ is slightly tilted with respect to the vertical direction when $B_{v}>0$, though we refer to $\mathbf{e}_{z}$ as the vertical direction for simplicity. There is no dependence of the turbulence along the $\mathbf{e}_{\|}$direction in the ideal interchange regime. The simulation equations and specific parameters are reported in Appendix A.

The simulated two-dimensional electrostatic potential, $\Phi(r, z, t)$, from quasi-steady-state ideal interchange turbulence is shown in Fig. 1 for a series of snapshots. One can reasonably divide this turbulence into two spatial regions. First, a mode region exists on the low-field side of the plasma profile, with typical vertical mode number $k_{z} \sim k_{\Delta}$. Second, a region at larger $R$ (Ref. 23) with lower plasma density and temperature is marked by intermittent structures termed blobs. $^{24}$

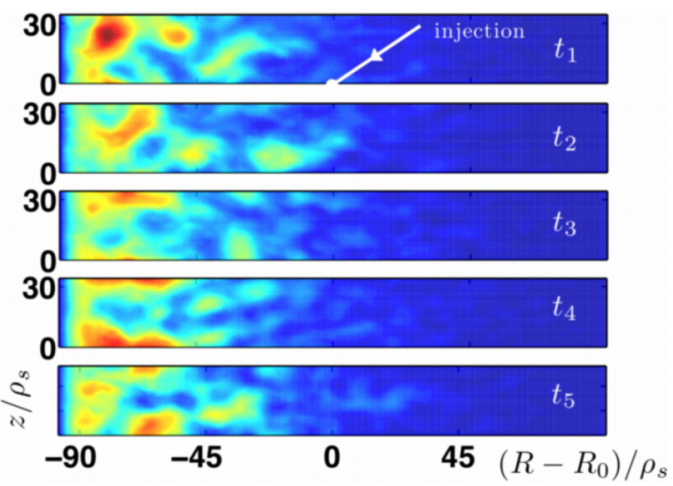

FIG. 1. Electrostatic potential $\Phi(r, z)$ in the nonlinear phase of ideal interchange turbulence in the SMT simulation described in Appendix A. These snapshots are at sequential times, $t_{i}$, such that $\Omega\left(t_{i+1}-t_{i}\right) \simeq 330$. 
Motivated by our goal of describing the dependence of suprathermal ion dynamics on the turbulence amplitude and by the fact that, as revealed by validation exercises, TORPEX turbulence tends to have a higher amplitude with respect to ion saturation current fluctuation in the experiment compared to the two-dimensional simulations, we modify a posteriori the turbulence simulation results, in order to vary the relative amplitude of turbulent fluctuations in which the ion motion is computed. The turbulent fluctuation amplitude is quantified by the parameter $\xi=e \tilde{\Phi} / \bar{T}_{e}$. Here, $\bar{T}_{e}$ denotes the electron temperature at the ion injection site averaged over both time and vertical coordinate, and $\tilde{\Phi}$ is the root mean square (rms) fluctuation amplitude of the turbulence. The value of $\xi$ is be varied as a parameter having an important effect on $\gamma_{R}$, by redefining the electric potential $\Phi$ as: $\bar{\Phi}+\xi(\Phi-\bar{\Phi}) / \xi_{0}$. Here, $\bar{\Phi}$ is the $t$ - and $z$-averaged radial electric potential profile. Note that $\xi$ decreases from the high-field side to the low-field side. The value of $\xi$ at $R=R_{0}, \xi_{0} \equiv \xi\left(R=R_{0}\right)$, is taken as the reference value $\xi_{0}$. In the SMT shown in Fig. 1, $\xi_{0} \sim 0.8$, and $0.8 \lesssim \xi / \xi_{0} \lesssim 1.2$, as a function of $\mathbf{e}_{R}$.

For comparison, we also study a slab magnetic field where ions move in $\left(\mathbf{e}_{x}, \mathbf{e}_{y}, \mathbf{e}_{z}\right)$ space, such that $\mathbf{B}=B_{0} \mathbf{e}_{y}$, with $B_{0}$ the same strength as $B\left(R_{0}\right)$ in the SMT. In the slab geometry, we use the same ideal interchange turbulence from simulations of TORPEX as a proxy for modes that occur in a slab geometry. ${ }^{25}$ Inserting the interchange-driven turbulence in the slab allows for a direct comparison between the slab and SMT magnetic geometries.

\section{SUPRATHERMAL ION TRAJECTORIES IN THE SIMPLE MAGNETIZED TORUS}

Injection conditions for suprathermal ions are inspired by the source used in TORPEX. ${ }^{7}$ A good estimate is an isotropic injection cone with opening angle $\sigma_{\alpha}=0.1 \mathrm{rad}$ and a Gaussian injection energy distribution. All of the simulations in this paper, unless otherwise stated, are performed with a point source. We denote with $\mathcal{E} \equiv M v_{0}^{2} /\left(2 \bar{T}_{e}\right)$ the average initial particle energy, normalized to the mean electron temperature at the injection site. The Gaussian injection distribution has a spread in velocity $\sigma_{v_{0}}=0.1 v_{0}$.

In the present manuscript, we consider an injection at the position $R=R_{0}$, a region where turbulence is transitioning from the mode region to the blob region (see top of Fig. 1). Thus, the ions enter the coherent mode region if they happen to move to the high-field side of the SMT. Otherwise, they are in the intermittent blob region if they happen to move to the low-field side. The amplitude of the turbulent fluctuations is larger in the mode region than in the blob region. A discussion addressing the dependence of ion beam spreading on the radial injection position is presented in Sec. IV B. Also, we focus on nearly parallel injection, such that the axis of the injection cone is directed along a field line. Perpendicular injection at a given energy tends to increase the Larmor radius of the particles and decrease the parallel velocity. Several suprathermal ion trajectories in a schematic of an SMT are shown in Fig. 2.

These ion trajectories are found by integrating the nonrelativistic equation of motion for charged particles,

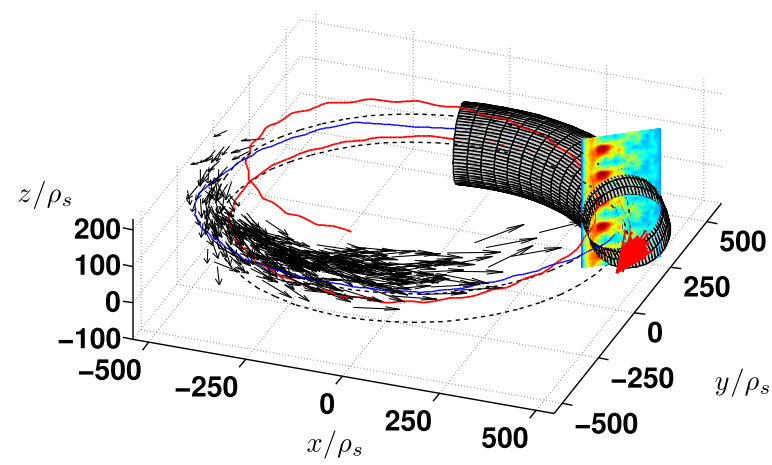

FIG. 2. Suprathermal ions in a schematic of TORPEX, with a section of the torus and a magnetic field line (dashed black line) indicated. Two suprathermal ion trajectories (red and blue solid lines) for a $\mathcal{E}=20$ injection are shown. The injection cone is indicated in red, while the black arrows indicate several ions at $t \Omega=130$. A snapshot of the electrostatic potential, $\Phi$, obtained from the simulations in Appendix A and used to integrate the ion trajectories, is shown with vertical periodicity of $\Delta$ at a poloidal crosssection.

$$
\frac{d \mathbf{v}}{d t}=\frac{q}{M}[\mathbf{E}+\mathbf{v} \times \mathbf{B}],
$$

for a time dependent SMT electric field $\mathbf{E}(\mathbf{R}, t)=-\nabla \Phi$ $(\mathbf{R}, t)$ and static magnetic field $\mathbf{B}(R)$, where $M$ is the mass and $q$ is the charge of the suprathermal ion. We remark that suprathermal ions are treated here as tracers, and therefore they do not influence the fields through Poisson's equation or Ampère's law. This fact, combined with the shorter timescales necessary for computing tracer trajectories in an open field-line configuration, allows us to use the full equation of motion without great numerical expense. We solve Eq. (1) with standard fourth-order Runge-Kutta time-stepping. The electric potential is interpolated using a bicubic algorithm in the perpendicular directions and a linear interpolation in time. The code has been checked for convergence with timestep and interpolation resolution, and a comparison with the Boris algorithm ${ }^{26}$ shows very good agreement. We ignore all collisions.

Our simulations use the full equation of motion, Eq. (1), but we find it very useful to consider particle drifts as we interpret the results. In the perpendicular plane, suprathermal ion trajectories in the SMT can be approximated by a combination of several elements: gyromotion, with suprathermal ion Larmor radius $\rho=\left|\mathbf{v}_{\perp} / \Omega\right|$ and frequency $\Omega=q B(R) / M$, the $\nabla \mathbf{B}$ drift, the $\mathbf{E} \times \mathbf{B}$ drift, and polarization drift. In the parallel direction, particle velocity is essentially unaffected since $k_{\|}=0$.

For SMT devices, drifts related to the curvature and radial gradient of the magnetic field, denoted as $\mathbf{v}_{\nabla \mathbf{B}}$, find a particularly simple expression,

$$
\mathbf{v}_{\nabla \mathbf{B}}=\mathbf{e}_{\|} \times\left(v_{\|}^{2} \mathbf{e}_{\|} \cdot \nabla \mathbf{e}_{\|}+\frac{v_{\perp}^{2}}{2 B} \nabla B\right) \frac{1}{\Omega}=\frac{1}{R}\left(\frac{v_{\perp}^{2}}{2}+v_{\|}^{2}\right) \frac{\mathbf{e}_{z}}{\Omega},
$$

so that this drift is purely in the vertical direction, $\mathbf{e}_{z}$. It is dominated by the curvature term $\left(v_{\|}^{2}\right.$ term) for large parallel injection energy. We note that the $\mathbf{v}_{\nabla \mathbf{B}}$ drift determines the 
displacement of the center of the ion beam in the vertical direction but also leads to a spreading of the beam in this direction due to the initial variation in particle energy and angle with respect to the field line. In the absence of a plasma, this drift causes ballistic spreading since it is exactly constant in time.

For the $\mathbf{E} \times \mathbf{B}$ drift, one must account for the gyroaveraged electric field, so that the gyroaveraged $\mathbf{E} \times \mathbf{B}$ velocity is $^{27,28}$

$$
\mathbf{v}_{\mathbf{E} \times \mathbf{B}}=\frac{1}{2 \pi} \oint \frac{\mathbf{E}\left(\mathbf{R}-\mathbf{v} \times \mathbf{e}_{\|} / \Omega\right) \times \mathbf{B}}{B^{2}} d \varphi,
$$

where $\varphi$ is the angle swept by the Larmor motion around the gyrocenter position. In practice, we compute the gyroaverage with a time average at the Larmor frequency. Consequently, if the suprathermal ion Larmor radius is significant compared to the scale of the turbulence, such that $k_{\perp} \rho \sim 1$, the gyroaverage significantly decreases the effective magnitude of the $\mathbf{E} \times \mathbf{B}$ drift velocity with respect to $k_{\perp} \rho \ll 1$. The timeaveraged turbulent steady-state electric field has a radial component causing a vertical $\mathbf{E} \times \mathbf{B}$ drift, while the fluctuating part of the electric field leads to alternating displacements in the radial and vertical directions. The size of these displacements is determined by the size and amplitude of the fluctuating vortex and blob-like structures in the turbulence.

The large fluctuations in the turbulent electric field cause significant velocity fluctuations in the perpendicular plane, which cause a polarization drift for the suprathermal ions. In the Lagrangian frame of the ion, these fluctuations can occur suddenly, in a fraction of the Larmor period. Therefore, they are able to break the conservation of the zeroth-order magnetic moment, $\mu=m v_{\perp}^{2} /(2 B) .^{30}$ In our simulations, we find that the non-conservation of $\mu$ is associated with increases or decreases in the Larmor radii of individual ions, such that an average increase in $\rho$ occurs over the ensemble, leading to heating of the ions. Our simulations confirm that larger fluctuation amplitudes cause faster heating regardless of the magnitude of the injection energy, and a study of the mass dependence of the heating shows that higher mass ions have a faster heating rate. As discussed later in Sec. V, this effect in the TORPEX device should be relatively minor.

The trajectories of individual ions in an SMT are displayed in Fig. 3(a), where we show the projection of two ion trajectories on the $(R, z)$ plane. These trajectories show the full particle motion, composed of gyromotion, vertical $\mathbf{v}_{\nabla \mathbf{B}}$ drift, and $\mathbf{v}_{\mathbf{E} \times \mathbf{B}}$ drift. Notably, the displacement of the gyrocenters in the radial direction is due only to the $\mathbf{v}_{\mathbf{E} \times \mathbf{B}}$ drift caused by turbulence. Figure 3(a) shows that radial $v_{\mathbf{E} \times \mathbf{B}}$ displacements are more significant for low energy particles due to gyro- and drift-averaging, as discussed in Sec. IV. The SMT trajectories can be qualitatively compared with slab trajectories in Fig. 3(b). In this geometry, vertical $\mathbf{v}_{\nabla \mathbf{B}}$ drifting clearly vanishes. One observes that turbulent radial displacements of the suprathermal ions are more frequent. This is particularly true in the case of particles with low energy. Additionally, an example of an energization event is shown in Fig. 3(b), with the increase of $\rho$ becoming apparent when the
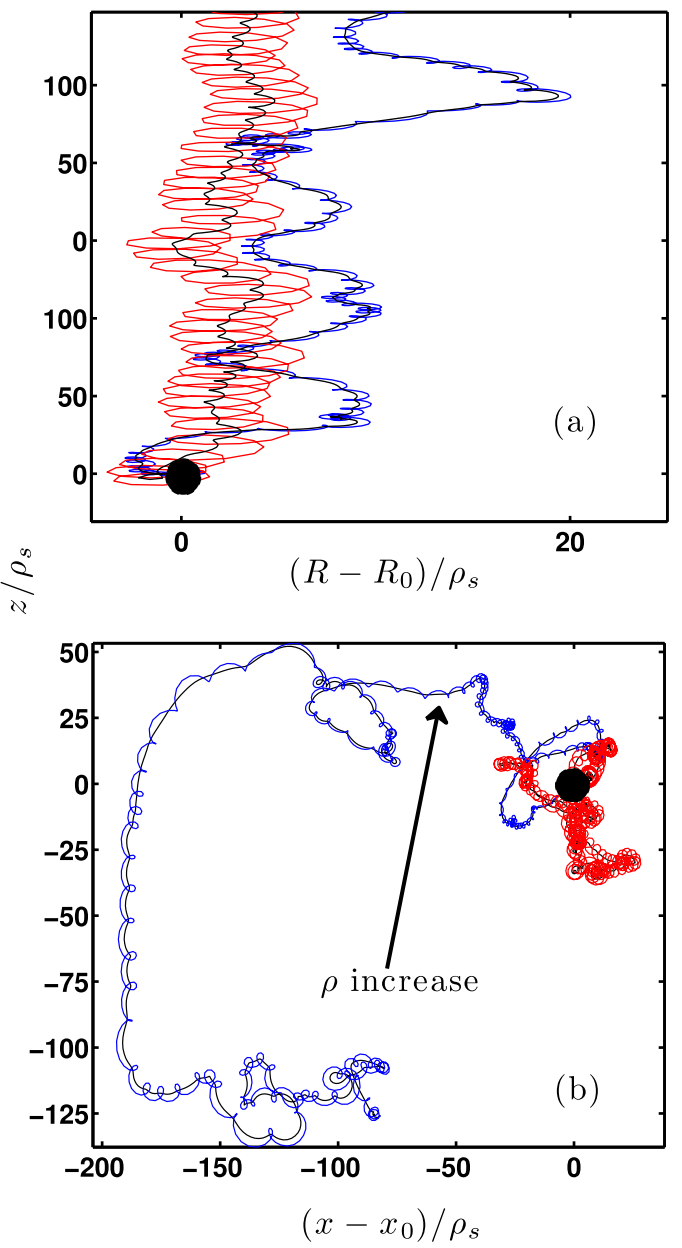

FIG. 3. Trajectories of lithium ions in the perpendicular plane with particle position and approximate gyrocenter positions (black) for high (red) and low (blue) energy. Initial suprathermal ion positions are at $R=R_{0}$ or $x=x_{0}$ and $z=0$ (black disk marker). In (a), an SMT with $\mathcal{E}=20$ (blue) and $\mathcal{E}=50$ (red) is used, while in (b), a slab with $\mathcal{E}=50$ (blue) and $\mathcal{E}=250$ (red) is used. The lower energy slab ion finishes with a larger gyroradius than its starting value because of the heating effect due to high-amplitude, fast variation of the electric field in the frame of the ion. The increase in $\rho$ occurs as the ion encounters a sharp gradient in the electric field at $\left(x \simeq-50 \rho_{s}, z \simeq 25 \rho_{s}\right)$.

ion enters the mode region. This is due to a sudden, impulsive acceleration event as the electric field changes rapidly in the frame of the cyclotronic ion motion. ${ }^{29,30}$ We emphasize that numerical convergence of this effect has been checked.

We now examine details of the dispersion of populations of ions injected nearly parallel to the magnetic field. For this purpose, we consider the role of single particle drifts in the dispersion process for both the SMT and slab magnetic geometries.

\section{SUPRATHERMAL ION BEAM DISPERSION IN THE SIMPLE MAGNETIZED TORUS}

For studying dispersion of suprathermal ions in turbulence, we use the variance

$$
\sigma_{R}^{2}(t) \equiv\left\langle\delta R^{2}\right\rangle
$$

of their radial displacements, $\delta R \equiv R(t)-R(0)$, where \langle\rangle is an ensemble average over many particle trajectories. 
Analogously, one can define the variances $\sigma_{z}$ and $\sigma_{\|}$, in the vertical and parallel directions, respectively. The results shown in the present paper have been evaluated by following the trajectories of $10^{4}$ suprathermal ions. Statistical convergence with particle number has been verified. We emphasize that the injection of the beam in our simulations is always at $R=R_{0}$, in the center of the computational box. The value of the turbulent fluctuation amplitude, $\xi$, on the low-field side tends to be lower than on the high-field side, so the choice of radial injection position can be significant. We argue later that the radial position can be parameterized by $\mathcal{E}$ and $\xi$.

Examination of $\sigma_{R}^{2}$ for the SMT at various injection energies $\mathcal{E}$ and turbulence levels $\xi$ reveals sequential phases for the $\gamma_{R}$ dispersion exponent for a range of experimentally relevant values of $\mathcal{E}$. We organize our discussion around the sequence of these phases, which tend to make a smooth transition from one into the next, as measured by the dispersion exponents. These are determined by fitting lines in log-log plots of $\sigma_{R}^{2}(t)$. The phases can be categorized as ballistic, interaction, and asymmetric, as shown in Fig. 4. These phases are now introduced and a few general comments given. In the following subsections, we then carefully consider dispersion in the radial direction, which is the main focus of the present work. Simple expressions to predict the duration and character of the three phases are deduced and tested with parameter scans in $\xi$ and $\mathcal{E}$.

The ballistic phase is a relatively brief period with $\gamma_{R} \simeq 2$ before the ions interact significantly with the turbulence and magnetic field. In the interaction phase that follows, depending on the injection energy and amplitude of the turbulence, suprathermal ion transport can be superdiffusive, $\gamma_{R}>1$, or subdiffusive, $\gamma_{R}<1$, depending on $\mathcal{E}$ and $\xi$. In the asymmetric phase, one observes that $\gamma_{R} \rightarrow 1$ and the dispersion is asymmetric. The late-time asymmetric phase is only relevant in the case where the interaction phase is subdiffusive.

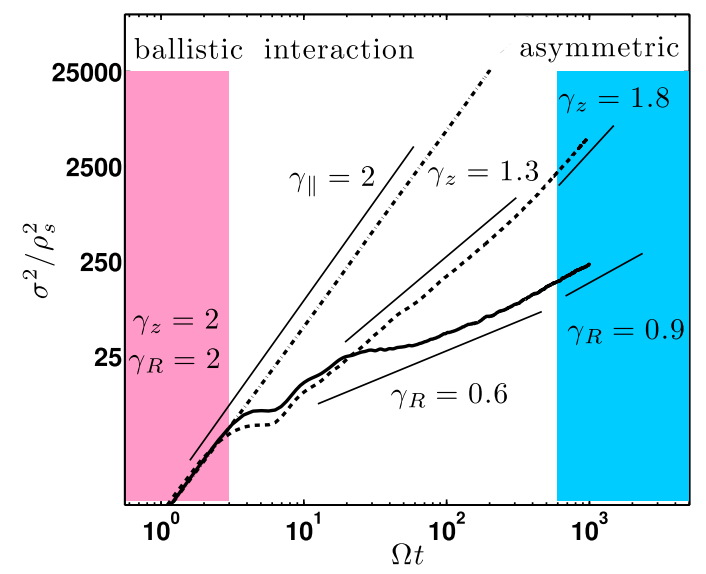

FIG. 4. Variance of displacements of suprathermal ions in the radial (solid curve), vertical (dashed curve), and parallel (dashed-dotted curve) directions for $\mathcal{E}=50$ and $\xi=0.8$ are shown. Dispersion exponents $\gamma$ are fitted with solid line segments, which have error of \pm 0.1 . An initial ballistic phase occurs (red-shaded region) with $\gamma_{R} \simeq \gamma_{z} \simeq 2$. This is followed by a fast transition to the turbulence interaction phase when $\gamma_{R}$ remains nearly constant. Later, a slower transition to the asymmetric phase (blue-shaded region) shows an increased value of $\gamma_{R}$. For the parallel direction, since there are no forces, $\gamma_{\|} \simeq 2$ always. The $z$-directed spreading also shows three phases in which the superdiffusion is due to $v_{\nabla \mathbf{B}}$.
In contrast, particle dispersion in $\mathbf{e}_{\|}, \sigma_{\|}$, is determined by initial spreading in parallel velocity and, since there are no forces in the parallel direction, it is essentially ballistic at all times. In $\sigma_{z}^{2}$, dispersion is roughly similar to $\sigma_{R}^{2}$, except that larger energies cause the $v_{\nabla \mathbf{B}}$ drift to become important relative to $v_{\mathbf{E} \times \mathbf{B}}$, which causes $\gamma_{z}$ to become superdiffusive.

On top of gyrocenter dispersion in the different phases, an oscillation in $\sigma_{R}^{2}$ and $\sigma_{z}^{2}$ is apparent for all $\mathcal{E}$ and $\xi$. This oscillation has amplitude comparable to $\rho^{2}$ and frequency $\Omega$. It occurs because the Larmor motions of the ions are synchronized at the injection point. This synchronization for point sources causes $\sigma^{2}$ to grow and shrink, a phenomenon that persists throughout our simulations. In fact, the relative phases of the Larmor motion do not decorrelate, since $B$ is constant except for small differences due to the $1 / R$ dependence of the magnetic field in the SMT. This oscillation amplitude remains approximately constant, and therefore it becomes relatively less important as the gyrocenter dispersion increases. An illustration of this phenomenon, showing growth in gyrocenter dispersion combined with a collective Larmor oscillation, is shown in Fig. 5. The collective oscillation can be removed by gyroaveraging in order to find the trend of the gyrocenter dispersion.

\section{A. Ballistic phase}

Suprathermal ions in the SMT have an initial ballistic spreading, analogous to the short ballistic transport phase in a typical collisional random walk observed recently by Huang et $a l .{ }^{35}$ in a neutral fluid. During this ballistic phase, particles move relatively unperturbed with respect to the initial velocity, unaffected by the turbulence, and therefore have a uniform motion. Due to the prescribed spread in initial velocities, uniform motion leads to a spatial dispersion with $\gamma_{R} \simeq 2$.

We now discuss the duration of this initial ballistic phase for the particles and gyrocenters. Let us first state that

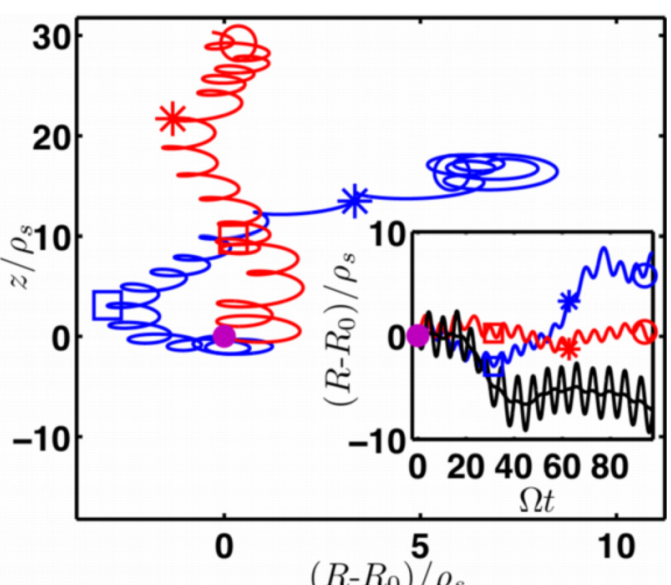

FIG. 5. Two particle trajectories (red and blue) for $\mathcal{E} \sim 35$ in the perpendicular plane. The inset shows the radial position as a function of time. Markers with the same shape (square, asterisk, and circle) mark equal time points. The separation between particle positions and between their gyrocenters is shown on the inset (black). Comparing two trajectories shows the tendency of the variance and mean of the perpendicular particle position to oscillate at the Larmor frequency, while at the same time, the variance of the gyrocenter positions grows steadily. 
the ballistic phase can, in principle, contain both a ballistic particle phase, ending at $t=\tau_{b a l}^{p}$ and a ballistic gyrocenter phase following the ballistic particle phase, with a duration $\tau_{b a l}^{g}$. Therefore, the ballistic phase may persist until $t=\tau_{b a l}$ $=\max \left\{\tau_{\text {bal }}^{p}, \tau_{\text {bal }}^{g}\right\}$.

The ballistic particle phase ends at: $\tau_{b a l}^{p} \simeq \min$ $\left\{2 / \Omega, m v_{\perp} / q|\mathbf{E}|\right\}$. During this period of time, particle velocity is not significantly affected by the magnetic field, nor by other forces, and therefore particles have a uniform linear motion that results in a ballistic spreading. These estimates compare very favorably with the simulations (see Figs. 4 and 10) where $v_{\perp}$ and $|\mathbf{E}|$ are measured.

During the ballistic gyrocenter phase, a particle gyrocenter moves at a constant velocity, with $\gamma_{R} \sim 2$ superimposed on a Larmor oscillation. This phase ends when the gyrocenter velocities change significantly due to encounters with structures in the turbulence. In the radial direction, the gyrocenter motion is given by the $\mathbf{v}_{\mathbf{E} \times \mathbf{B}}$ drift. Therefore, in order to estimate $\tau_{b a l}^{g}$ one must determine the time for a change in the gyrocenter speed to be comparable to its initial speed, such that

$$
\frac{\Delta v_{\mathbf{E} \times \mathbf{B}, R}}{v_{\mathbf{E} \times \mathbf{B}, R}} \sim 1 .
$$

This corresponds to the time taken for the particle to enter a region where the electric field changes significantly compared to its initial value.

A test ion can encounter a change in the electric field in two ways, depending on whether ions move through the field faster than turbulent field structures move past the ions. First, if the particle moves through the electric field faster than the field changes in the lab frame, the time necessary for the particle to experience a different gyroaveraged electric field is given by $\tau_{m}=\lambda_{c} / v_{g}$, with $\lambda_{c}$ indicating a fraction of the typical scale length of the electric field, and $v_{g}$ the typical gyrocenter velocity. Conversely, the electric field may change in the lab frame more quickly than the ion moves, and then $\tau_{b a l}^{g}$ is the Eulerian correlation time of the turbulence, $\tau_{c}$. The duration of the ballistic gyrocenter phase corresponds to the minimum of the two: $\tau_{\text {bal }}^{g}=\min \left\{\tau_{m}, \tau_{c}\right\}$.

Regarding the evaluation of $\tau_{m}$ for the turbulence we are considering, the gyrocenter velocity $v_{g}$ can be dominated by either $v_{\mathbf{E} \times \mathbf{B}}$ or $\mathbf{v}_{\nabla \mathbf{B}}$, whichever is larger. Therefore,

$$
\tau_{m} \sim\left\{\begin{array}{ccc}
\lambda_{c} / v_{\mathbf{E} \times \mathbf{B}} & \text { if } & v_{\mathbf{E} \times \mathbf{B}}>v_{\nabla \mathbf{B}} \\
\lambda_{c} / v_{\nabla \mathbf{B}} & \text { if } & v_{\mathbf{E} \times \mathbf{B}}<v_{\nabla \mathbf{B}}
\end{array} .\right.
$$

As for $\tau_{c}$, this is the Eulerian correlation time, measured as the decay time of the exponential fit to the autocorrelation function for the plasma potential in time. Our value of $\tau_{c}$ from the nonlinear simulations is similar to the inverse of the linear growth rate of the ideal interchange mode, ${ }^{15}$ $\sqrt{2} c_{s} / \sqrt{R L_{p}}$. In all of our simulations, we note that the value of $\tau_{b a l}^{g}$ taken from this estimate is comparable to $\tau_{b a l}^{p}$. Thus, we do not observe any substantial ballistic gyrocenter phase, though it may be important in other contexts with weaker turbulence. Considering the dispersion in the vertical direction, we note that the ballistic phase for $\gamma_{z}$ has the same behavior as for $\gamma_{R}$.

\section{B. Interaction phase}

Following the ballistic phase, suprathermal ions interact strongly with the turbulence. The beam dispersion then enters a phase where $\gamma_{R}$ remains nearly constant before the radial boundaries change the dispersion significantly. We identify this as the interaction phase, since suprathermal ion interaction with turbulence is most important to their dispersion. One cause of the end of the interaction phase is that a significant number of particles reach the vessel wall at the high-field side. This is observed after superdiffusive spreading for small $\mathcal{E}$. On the other hand, for the subdiffusive cases, $\gamma_{R}$ grows as a significant number of ions move into the mode region, where turbulence properties are different compared to the injection point. We describe this in Sec. IVC as the asymmetric phase.

Now, for the interaction phase, we are interested in computing the nondiffusive values of $\gamma_{R}$ as a function of $\xi$ and $\mathcal{E}$. There are essentially three mechanisms that determine the value of $\gamma_{R}$ during the interaction phase, which we analyze in detail based on drift approximations. First, the turbulence in $\Phi$ gives $v_{\mathbf{E} \times \mathbf{B}}$ in the radial direction, which is the source of radial dispersion in our model. In the limit of $k_{\perp} \rho \rightarrow 0$ and $\nabla \mathbf{B} \rightarrow 0$, this turbulent dispersion is superdiffusive because some structures in the turbulence are relatively static and have large amplitudes relative to the background profile, allowing ions to move large distances through the structures. ${ }^{36}$ However, if the fluctuations are reduced below a certain level, $\gamma_{R}$ drops dramatically because the amplitude of the vortex structures is too small for the structures to form connected velocity streamlines between the center and edge of the plasma. This is simply a topological constraint set by the amplitude of the turbulent fluctuations.

Second, larger values of $k_{\perp} \rho$ lead to substantial changes in the particle dynamics. In fact, a large Larmor radius can gyroaverage the electric field fluctuations and reduce the effective radial $v_{\mathbf{E} \times \mathbf{B}}$ drift. As a consequence, gyroaveraging reduces the number and size of the steps in the radial (and vertical) direction. We expect, therefore, that for $k_{\perp} \rho \gtrsim 1$, radial dispersion of the particles has a smaller $\gamma_{R}$ than for $k_{\perp} \rho<1$. We note that $\rho \sim v_{\perp} / \Omega$, where $v_{\perp}$ can be estimated as the sum of the $\nabla \mathbf{B}$ drift, the turbulence-induced $\mathbf{E} \times \mathbf{B}$ drift, and the initial velocity.

Third, the vertical drift velocity $\mathbf{v}_{\nabla \mathbf{B}}$ has an important effect on the suprathermal ion dynamics. In fact, if the vertical motion is sufficiently fast, an effective drift-average of the electric field fluctuations reduces the radial dispersion, making it subdiffusive. This can occur if the time required for an ion to traverse a turbulence wavelength vertically is significantly smaller than the radial traversal time. This ratio can be estimated as follows. Let $\tau_{R}$ be the time required to move radially across the structure, such that

$$
\tau_{R} \sim L_{R} / v_{\mathbf{E} \times \mathbf{B}, R},
$$

where $L_{R}$ is the radial extent of the vortex, which has been estimated $^{37}$ as $L_{R} \sim \sqrt{L_{p} / k_{\Delta}}$. Similarly, we define

$$
\tau_{z} \sim L_{z} /\left(v_{\nabla \mathbf{B}}+v_{\mathbf{E} \times \mathbf{B}, z}\right)
$$


as the time required to cross a vortex of size $L_{z} \sim 1 / k_{\Delta}$ due to the vertical velocity. The curve

$$
\frac{L_{R}\left(v_{\nabla \mathbf{B}}+v_{\mathbf{E} \times \mathbf{B}, z}\right)}{L_{z} v_{\mathbf{E} \times \mathbf{B}, R}} \simeq \chi
$$

defines a region where the ions are more likely to complete radial steps before vertical drift-averaging makes radial steps less likely.

The numerical parameter $\chi \simeq 5$ is the approximate value for a transition to subdiffusive values of $\gamma_{R}$ for all values of $\xi$ tested. We note that in the subdiffusive regime, particles move while maintaining nearly the same radial positions. Therefore, because of the $\phi$-periodic boundary condition in the vertical direction, particles move through an electric field that can be decomposed in Fourier modes, with $k_{z}=k_{\Delta}$ or multiples thereof. This causes a radial $\mathbf{E} \times \mathbf{B}$ drift, which averages towards zero amplitude during the trajectory, since it will produce a leftward displacement followed by a rightward displacement with a similar amplitude. This explanation for subdiffusion can be compared with that given in Sánchez et al ${ }^{38}$ for turbulent eddies in a shear flow.

In Fig. 6(a), we plot the values of $\gamma_{R}$ in the interaction phase as obtained from a large number of simulations in the SMT. The solid curve in Fig. 6(a) shows the boundary for $k_{\Delta} \rho=2$. Outside of this boundary, gyroaveraging gradually reduces $\gamma_{R}$ from superdiffusive to subdiffusive values. The condition for $\mathbf{v}_{\nabla \mathbf{B}}$ averaging, given by Eq. (8), is also

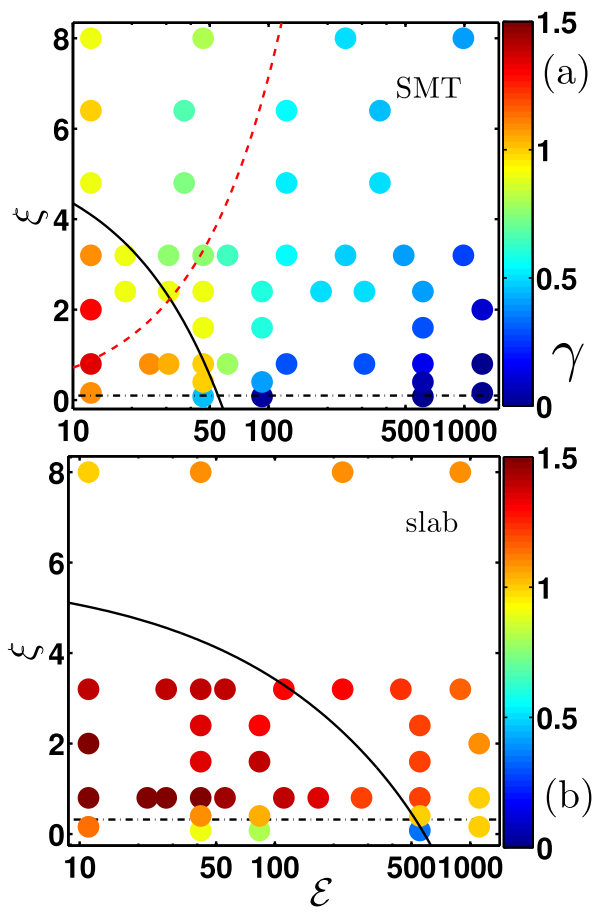

FIG. 6. Dispersion exponents $\gamma_{R}, \gamma_{x}$ (colored dots) in the interaction phase are presented in the $(\mathcal{E}, \xi)$ space for the SMT (a) and slab (b). Error on the value of $\gamma$ is \pm 0.1 . For $\xi<0.3$ (dashed-dotted horizontal lines), the turbulent fluctuations are too small for connected radial streamlines to form, therefore $\gamma \rightarrow 0$ abruptly. Above the $k_{\Delta} \rho=2$ (solid black curves) gyroaveraging reduces $\gamma$. In the SMT case, drift averaging from Eq. (8), is indicated by the dashed red curve. To the right of this curve, drift averaging reduces $\gamma_{R}$. displayed in Fig. 6(a) with a dashed curve, confirming the reduction of the suprathermal ion dispersion rate due to drift-averaging. Finally, the $\xi=0.3$ boundary, for which connected streamlines do not form, is indicated by a dasheddotted line. The reduction of $\gamma_{R}$ related to this topological effect occurs more sharply than the other effects described previously.

For comparison, we also display $\gamma_{R}$ values in the slab case in Fig. 6(b), for which drift-averaging due to $\mathbf{v}_{\nabla \mathbf{B}}$ is not present. As expected, $\gamma_{x}$ values in the slab are generally larger than in the SMT. Gyroaveraging effects are still apparent, as shown by the curved boundary at $k_{\perp} \rho=2$ in Fig. 6(b). Moving past this boundary reduces superdiffusive values of $\gamma_{x}$ to diffusive values for $k_{\Delta} \rho>2$. A sharp reduction of $\gamma_{x}$ for $\xi<0.3$ is also shown, explained by a change in topology of the streamlines, as discussed for the SMT case.

To summarize the results of the interaction-phase values of $\gamma_{R}$ and $\gamma_{x}$ as a function of $\xi$ and $\mathcal{E}$, one must first consider that, for sufficiently large turbulent fluctuations, large and persistent $\mathbf{E} \times \mathbf{B}$ drifts cause superdiffusive dispersion. Gyroaveraging of $v_{\mathbf{E} \times \mathbf{B}}$ drifts, an effect present in both the slab and the SMT, tends to decrease both $\gamma_{R}$ and $\gamma_{x}$. For the SMT, $v_{\nabla \mathbf{B}}$ leads to an additional drift-averaging which reduces $\gamma_{R}$ with respect to the slab $\gamma_{x}$ case.

We now discuss the injection-position dependence of fast-particle spreading in the interaction phase. Provided that the parameters $\xi$ and $\mathcal{E}$ are evaluated with the temperature and fluctuation potential at the injection position, we assert that $\gamma_{R}$ depends on $\xi$ and $\mathcal{E}$ according to Fig. 6 independently of radial injection positions. Thus, the value of $\gamma_{R}$ depends on $\xi$ and $\mathcal{E}$, while it appears to depend on the injection position $R$ only because $\xi$ and $\mathcal{E}$ depend on $R$. This makes the results of Fig. 6 of general interest.

We test our assertion by performing two sets of simulations. We consider the fast ions that, for the injection position $R=R_{0}$, are characterized by $(\mathcal{E} \simeq 46, \xi \simeq 0.4)$ and by $\left(\mathcal{E} \simeq 250, \xi \simeq 3.2\right.$ ). While keeping $v_{0}$ constant, we change the radial injection position. In particular, we consider the radial injection positions $\left(R-R_{0}\right) / \rho_{s} \simeq \pm 35$. This variation in $R-R_{0}$ causes $\bar{T}_{e}$ to change by a factor of two in either direction, while $\xi$ remains roughly constant. Thus, at the radial positions $\left(R-R_{0}\right) / \rho_{s} \simeq\{-35,0,35\}$, the low-energy ions are injected with $\mathcal{E} \simeq\{23,46,92\}$ and $\xi \simeq 0.4$, while the high-energy ions are injected with $\mathcal{E} \simeq\{125,250,500\}$ and $\xi \simeq 3.2$. The simulated values are $\gamma_{R} \simeq\{1.0,1.0,0.5\}$ for the low-energy ions and $\gamma_{R} \simeq\{0.6,0.5,0.4\}$ for the high-energy ions, as shown in Fig. 7. These agree well with the interpolated values of $\gamma_{R}$ in Fig. $6, \gamma_{R} \simeq\{1.1,1.0,0.4\}$ and $\gamma_{R} \simeq\{0.6,0.5,0.4\}$, respectively.

Finally, we briefly describe spreading along the $z$ direction. In the SMT configuration, the effect of the $\mathbf{E} \times \mathbf{B}$ turbulent velocity is superimposed on ballistic spreading in $\mathbf{e}_{z}$ due to the $\mathbf{v}_{\nabla \mathbf{B}}$ drift. This ballistic spreading is related to the injection of particles with different energies. As a matter of fact, the simulations show that $1<\gamma_{z} \leq 2$, with $\gamma_{z} \rightarrow 2$ at high $\mathcal{E}$, i.e., in the regime where the $\mathbf{v}_{\nabla \mathbf{B}}$ velocity dominates over the turbulent $\mathbf{E} \times \mathbf{B}$. On the other hand, in the slab configuration, $\gamma$ values in the $x$ and $z$ direction are approximately the same. 


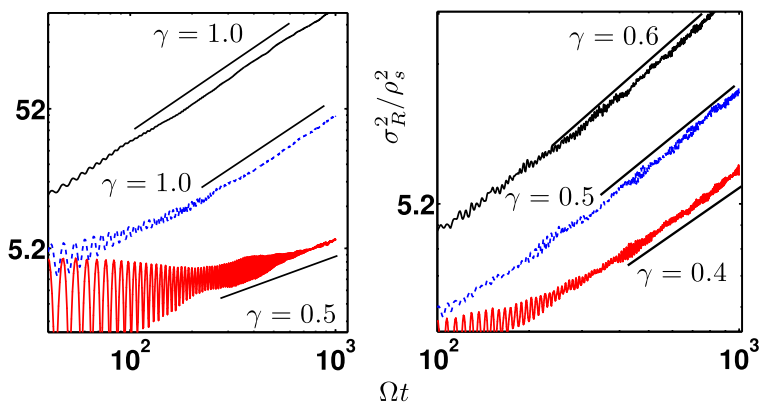

FIG. 7. Spreading of fast ions in the radial direction for three different values of $\left(R-R_{0}\right) / \rho_{s} \simeq\{-35,0,35\}$, shown with solid black curves, dashed blue curves and dotted red curves, respectively. Values of $\gamma_{R}$ are indicated with best-fit lines. The low-energy ions are on the left panel, corresponding to $\mathcal{E} \simeq\{23,46,92\}$ and $\xi \simeq 0.4$, while the high energy ions are on the right panel, corresponding to $\mathcal{E} \simeq\{125,250,500\}$ and $\xi \simeq 3.2$.

\section{Asymmetric phase}

A third phase for the spreading rate is apparent if, during the interaction phase, one observes subdiffusive radial spreading of the particles: $\gamma_{R}<1$. This phase typically shows an increase of the $\gamma_{R}$ exponent with respect to the interactionphase value, to the point that $\gamma_{R} \simeq 1$. However, this phase is not precisely diffusive but rather asymmetric, such that the leftward dispersion into the mode region is superdiffusive, while the rightward dispersion into the blob region remains subdiffusive. The mean value of these two behaviors gives $\gamma_{R} \simeq 1$. This asymmetry is most easily seen in the probability distributions of displacements shown in Fig. 8.

The asymmetric phase appears after the beam has spread enough to sample a larger radial neighborhood of the simulated turbulence. This sampling has important effects. In fact, we note that both $\xi$ and $\mathcal{E}$ depend on the radial position, since $\bar{T}_{e}=\bar{T}_{e}(R)$. As the beam spreads, therefore, $\mathcal{E}$ and $\xi$ can change significantly with respect to their values at the injection position. Our previously mentioned numerical tests show that this implies a change in $\gamma_{R}$, in accordance with Fig. 6. Radial dispersion can therefore be asymmetric due to asymmetric variation in $\xi$ and $\mathcal{E}$.

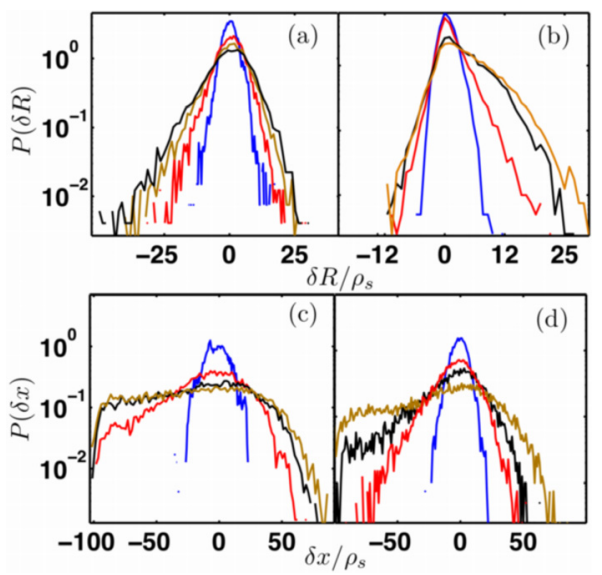

FIG. 8. Probability density (PDF) of suprathermal ion displacements in the direction of the density gradient for the SMT: (a) $\mathcal{E}=50$ and (b) $\mathcal{E}=250$ injection, and slab: (c) $\mathcal{E}=50$ and (d) $\mathcal{E}=250$ injection. The color of the PDF changes from blue-red-black-brown, in evenly spaced time increments for $0 \leq \Omega t \leq 1000$.
We also remark that in simulations showing $\gamma_{R}>1$ or $\gamma_{x}>1$, during the interaction phase, the beam always strikes the radial boundary before a third phase is apparent. It should be remembered, however, that this superdiffusion is already asymmetric, biased towards the mode region where $\bar{T}_{e}$ is higher, as shown in Fig. 8. For large injection energies, $\gamma_{R}$ remains subdiffusive for very long times, as the ions do not move into the mode region in significant numbers. On the other hand, as seen in Fig. 8(b), particle radial displacements are asymmetric towards the low-field side because of the dependence of $\rho$ on $B(R)$.

\section{INITIAL COMPARISONS WITH EXPERIMENTAL DATA}

Having compiled a basis of results for understanding suprathermal ion transport in turbulent plasmas for the SMT configuration, we discuss how our theory can be applied to the interpretation of TORPEX experimental data and show an exploratory comparison between experiments and simulations.

The TORPEX source emits suprathermal ions with energies in the range $0.1<\mathcal{E} \bar{T}_{e}<1 \mathrm{keV}$. For $\bar{T}_{e} \simeq 5-15 \mathrm{eV},{ }^{3}$ this results in suprathermal ion energy ratios $10 \lesssim \mathcal{E} \lesssim 200$, which are relevant to fusion-related neutral beam experiments. ${ }^{39}$ We emphasize that our study has focused on injection at $R=R_{0}$. We remark that it is possible to vary $\bar{T}_{e}$ by changing the injection location. Since the parameters of interest, $\xi$ and $\mathcal{E}$, are dependent on $\bar{T}_{e}$, and therefore on the injection position, it follows that the radial position of the injection is an important control parameter for suprathermal ion dynamics.

In general, we note that a detailed experimental comparison with our comprehensive numerical analysis of suprathermal ion behavior is fundamentally limited by the SMT for a number of reasons. The finite vertical size of the SMT limits the field-line connection length as well as the vertical distance through which ions drift with the $\mathbf{v}_{\nabla \mathbf{B}}$ and $\mathbf{v}_{\mathbf{E} \times \mathbf{B}}$ velocities before colliding with the vessel. Similarly, the radial boundaries of the SMT are a limiting factor for the measurement. The radial dimension of the SMT tends to be the limiting factor in experimental comparisons of superdiffusive ions at low injection energies, while the vertical dimension limits comparisons for larger $\mathcal{E}$. Moreover, the signal-to-noise ratio of the detector limits the distance at which the detector can be placed with respect to the source.

Recent measurements have provided suprathermal ion current profiles at a single toroidal separation between source and detector. ${ }^{3}$ As an example, Fig. 9 (left column) shows experimental profiles of suprathermal ion currents in the absence (top row) and presence of plasma (bottom row). The suprathermal ions have energy $E=88 \mathrm{eV}$, and the detector is toroidally separated by $40 \mathrm{~cm}$ from the source. The measurements show that the beam is spread by the interaction with the turbulent plasma. The spreading of the suprathermal ion beam can be compared with our simulations. For this purpose, we create a synthetic diagnostic for ion current profiles that mimics the experimental technique. The synthetic ion current is composed of the integrated product of the ion density and velocity in the perpendicular plane at a specified 

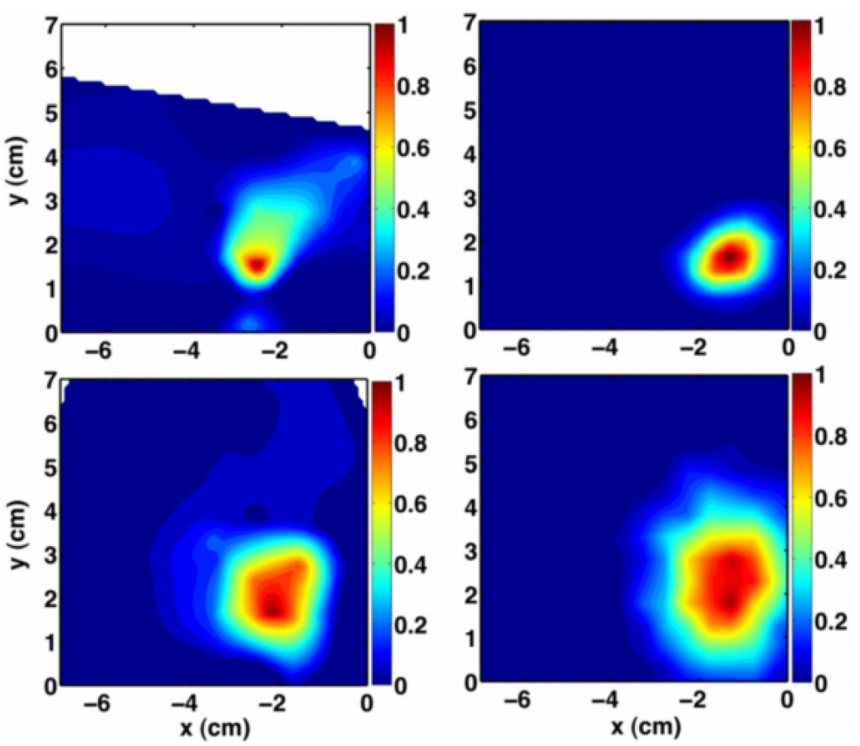

FIG. 9. Experimental (left) and synthetic (right) suprathermal ion current diagnostic in a poloidal cross section $40 \mathrm{~cm}$ from the injection point. The energy is $88 \mathrm{eV}(\mathcal{E} \simeq 40)$ and $\xi \simeq 0.6$. The increase in spreading in the presence of plasma (bottom) compared to no plasma (top) is comparable for both simulation and experiment. Colors represent ion current magnitude in arbitrary units.

toroidal location. The poloidal resolution of the synthetic detector is set to match the resolution of the real detector.

The initial conditions for injection are computed from the measured current profile without plasma. This allows the injection angle and spreading in energy to be tuned for agreement before activating the plasma. The simulated suprathermal ion source for this experimental comparison is a Gaussian distribution with width similar to the experimental beam opening.

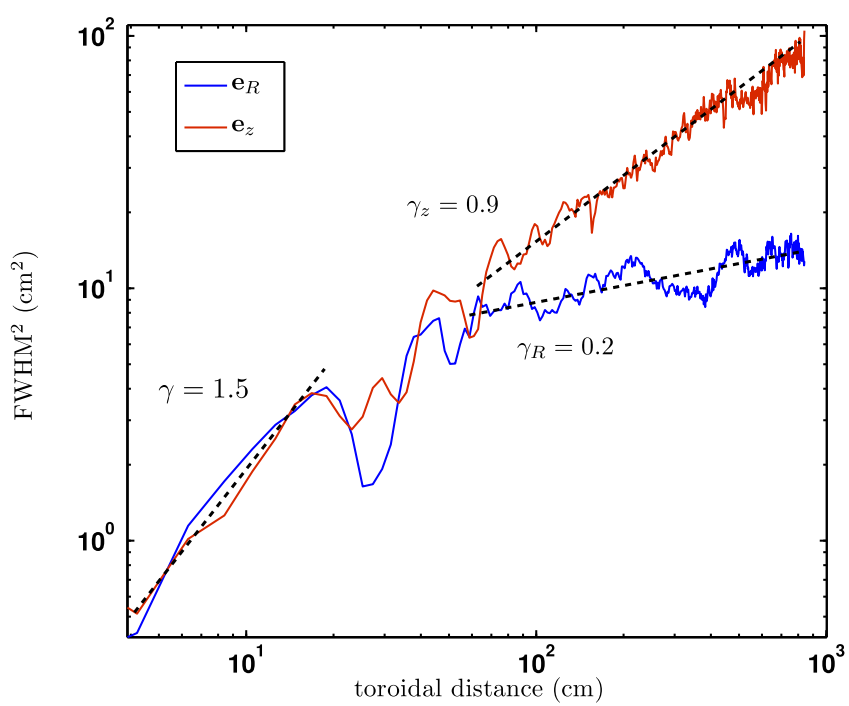

FIG. 10. Radial (solid blue) and vertical (dashed black) squared full width at half maximum (in $\mathrm{cm}^{2}$ ) for $\mathcal{E} \simeq 40$ in a TORPEX ideal interchange turbulence simulation, tuned to the experiment with $\xi \simeq 0.6$. At $40 \mathrm{~cm}$ separation between source and detector, the interaction phase has begun. This is where the experimental ion current profile is taken for Fig. 9. Straight lines show approximations to the value of $\gamma_{R}$ and $\gamma_{z}$. The value of $\gamma$ from FWHM is systematically smaller than that from $\sigma^{2}(t)$.
As Fig. 9 shows, radial and vertical spreading of the profile increases by a similar amount for both experiment and simulation for a turbulent, ideal interchange mode plasma. This experimental comparison corresponds to $\mathcal{E} \sim 40$ and $\xi_{0} \sim 0.6$, with the simulation calibrated to both the fluctuations and the position of the peak values in the floating potential and the measured temperature profile, respectively. Figure 10 shows the full width at half maximum (FWHM) measurement from the synthetic diagnostic as a function of the toroidal distance between source and detector. This measurement is a proxy for $\sigma^{2}(t)$ for determining the value of $\gamma$. The value of $\gamma$ in the FWHM is systematically smaller than the value from $\sigma^{2}(t)$, since the FWHM does not see the tails of the distribution of ion positions. Nevertheless, when the TORPEX experiment is able to achieve a large number of toroidal measurements, it should be able to distinguish between subdiffusive and superdiffusive $\gamma$.

We note that in the model, we have ignored collisions with background electrons and ions. Our estimate of the beam spreading due to Coulomb collisions is at least one order of magnitude smaller than that due to turbulent transport.

\section{SUMMARY AND CONCLUSIONS}

Our numerical study of suprathermal ion transport driven by ideal interchange mode turbulence inside a simple magnetized torus configuration provides a comprehensive description of the interplay of fundamental aspects influencing charged-particle motion in electric and magnetic fields. In this turbulence, suprathermal ion transport is generally nondiffusive, as characterized by the transport exponent, $\gamma_{R} \neq 1$. Moreover, the value of $\gamma_{R}$ changes with time after injection. A short ballistic phase is followed by an interaction phase with either subdiffusive, diffusive or superdiffusive character. Generally, $\gamma_{R}$ is larger towards the high-field side than on the low-field side. Thus, for subdiffusive cases, the value of $\gamma_{R}$ tends to increase, with respect to the interaction phase, and transition into an asymmetric phase after a certain amount of time, i.e., when the suprathermal ions have spread sufficiently to sample the higher amplitude turbulence closer to the plasma source.

In the interaction phase, suprathermal ion dynamics depend on the injection energy and the fluctuation amplitude of the turbulent electric field, given by the parameters $\mathcal{E}$ and $\xi$, respectively. This dependence results essentially from the competition of three effects. First, the $\mathbf{E} \times \mathbf{B}$ drift motion tends to cause superdiffusion due to long-distance transport by largescale turbulent vortices. However, larger $v_{\perp}$ tends to increase the suprathermal ion Larmor radius and slow the spreading by decreasing the gyrocenter $\mathbf{E} \times \mathbf{B}$ drift velocity. Gyroaveraging tends to reduce $\gamma_{R}$ to diffusive values. Thirdly, in constrast to gyroaveraging, vertical drift-averaging forces $\gamma_{R} \rightarrow 0$ and $\gamma_{z} \rightarrow 2$. This analysis assumes that the fluctuation amplitude is large enough to create vortices with significant radial extension relative to the device dimensions. If there are few connected streamlines, there is very little transport due to turbulence.

Let us confront the important general question of how well a turbulent diffusion model can represent the spreading 
of the ion beam. A diffusive model is often used to describe suprathermal ion transport in fusion plasmas, ${ }^{31}$ though nondiffusive models have been successful in many cases. ${ }^{32,33}$ Diffusion is sometimes sufficient to describe suprathermal ion transport over certain time and spatial scales, since a nondiffusive process may always be linearized as diffusion. Models with spatial dependence in the diffusion coefficient have been used in the plasma physics literature ${ }^{34}$ as an alternative to nondiffusive dispersion.

For comparison with the usual diffusive assumption, we consider whether our effective suprathermal ion diffusivities are roughly in agreement with measurements of particle diffusivity computed as $D_{I}=\Gamma_{n} / \partial_{R} n$, where $\Gamma_{n}$ is the radial particle flux. For the ideal interchange mode in the TORPEX plasma, the diffusivity $D_{I}$ can be found in Ref. 15:

$$
D_{I}=\alpha \frac{1}{k_{\Delta}} \sqrt{\frac{2 \bar{T}_{e} L_{p}}{m_{i} R_{0}}}\left(1-\frac{10 L_{p}}{3 R_{0}}\right)^{3 / 2}
$$

where $\alpha \sim 0.34$ and $L_{p}$ is the pressure gradient scale length.

Using particle tracking one can find $\sigma^{2}(t)$ and make a linear approximation to a diffusivity (referred to as the effective diffusivity $\left.D_{i o n}\right)$ such that $\sigma^{2}(t) \sim D_{\text {ion }} t$. At $\xi=\xi_{0}$, for low energy ions at $\mathcal{E}<8$ (approaching the plasma ion temperature), $D_{\text {ion }}^{l} \simeq D_{I}$ when making the linear approximation for a superdiffusive dispersion. For $\mathcal{E} \sim 100, D_{i o n}^{h} \simeq D_{I} / 30$ because the dispersion is subdiffusive, with $\gamma<1$. Therefore, the suprathermal ion dispersion in an SMT is significantly overestimated by the diffusive interchange mode estimate. When $\mathcal{E}>100$, the diffusive estimate can be more than two orders of magnitude too large.

Available data from the TORPEX device indicate that the magnitude of suprathermal ion dispersion is consistent with simulations at a single point at the beginning of the interaction phase. Our work indicates the interest of measuring the different phases of the transport. In particular, an experiment should be attempted to measure $\sigma^{2}$ from the ballistic to the interaction phase, as was done for fluids recently by Huang et al.$^{35}$ For this measurement, resolution in the toroidal direction is required, which will soon be possible in TORPEX with a toroidally moving source. Based on our earlier discussion of the ballistic phase, the toroidal separation of the source from the detector, $L_{b}$, at the end of this phase is

$$
L_{b}=\tau_{b a}\left\langle v_{0,||}\right\rangle
$$

On the other hand, measuring a change in $\gamma_{R}$ into the asymmetric phase is difficult because most of the ion beam tends to exit the plasma before this transition is well-resolved.

We note that the turbulent heating effect mentioned in Sec. IV may be observable in experiments. However, our estimates show that the magnitude of the heating increases the overall temperature of the suprathermal ions by only $T_{i} \sim 1 \mathrm{eV}$ before they reach the parallel distance at which field lines contact the vacuum vessel. TORPEX is not currently equipped to measure the ion temperature to this precision, but the mechanism we observe may be an explanation for the $T_{i} \sim 1 \mathrm{eV}$ measurement in TJ-K. ${ }^{41}$
We believe that the theoretical framework we have developed here may be relevant to measurements reported elsewhere for a linear, slab-like, machine. Using a suprathermal ion diagnostic similar to the TORPEX diagnostic, Zhou et al. ${ }^{16}$ took several measurements along the parallel coordinate for the LAPD device. These LAPD measurements show ballistic spreading, with $\gamma_{x} \sim 2$ at a separation between source and detector of $150 \mathrm{~cm}$, followed by subdiffusive $\mathrm{e}^{40}$ spreading. This is similar to the behavior seen in some of our simulations, between the ballistic phase and the interaction phase, as in Fig. 10.

The interplay of fundamental influences from gyromotion and curvature drift, which determine the transport of suprathermal ions in the SMT, is also present in fusion confinement configurations. Investigations of suprathermal ions in the fusion context could use these results as a basis. Radial constraint of transport due to zonal flows associated with iontemperature gradient (ITG) turbulence ${ }^{42}$ or other velocity shearing mechanisms may lead to similar subdiffusive tendencies as we find to be caused by curvature drift in the SMT. We view $\mathbf{E} \times \mathbf{B}$ smoothing due to low- $k$ modes, as demonstrated for ITG, ${ }^{33}$ to be analogous to the curvature drift averaging noted here. This qualitative connection could be pursued in future work. The relevance of suprathermal ions in astrophysics and space physics phenomena such as cosmic rays and solar flares ${ }^{11-13,43}$ is also well-known. The general rules found here are relevant in those contexts as well.

\section{ACKNOWLEDGMENTS}

We appreciate especially helpful discussions with Alice Burckel, Mattia Albergante, Stephan Brunner, Mark Gilmore, Jonathan Graves, Federico Halpern, Joaquim Loizu, and Christian Theiler. This work was supported by NSF-IRFP Award No. 0853498 and by the Swiss National Science Foundation.

\section{APPENDIX A: DRIFT-REDUCED BRAGINSKII MODEL FOR INTERCHANGE MODE TURBULENCE}

Drift-reduced Braginskii equations ${ }^{20,44}$ are used for plasma modeling in many contexts (see e.g., Refs. 2, 5, and 45). In the present paper, we consider $k_{\|}=0$ turbulence, $T_{i} \ll T_{e}$, and electrostatic fields. We assume $B_{v} \ll B_{\phi}$ and constant curvature. Bohm's boundary conditions are used for parallel flow at the sheath edge. The Boussinesq approximation for the polarization drift ${ }^{46}$ is taken as

$$
\nabla \cdot\left(\frac{n m_{i}}{e B} \frac{d \nabla \Phi / B}{d t}\right)=\frac{n m_{i}}{e B^{2}} \frac{d}{d t} \nabla^{2} \Phi
$$

With these assumptions, the equations for the line-integrated density, $n(r, z)$, potential, $\Phi(r, z)$ and electron temperature $T_{e}(r, z)$ are

$$
\begin{aligned}
\frac{\partial n}{\partial t}= & \frac{c}{B}[\Phi, n]+\frac{2 c}{e R_{0} B_{0}}\left(n \frac{\partial T_{e}}{\partial z}+T_{e} \frac{\partial n}{\partial z}-e n \frac{\partial \Phi}{\partial z}\right)+D \nabla^{2} n \\
& -\frac{\sigma n c_{s}}{R_{0}} \exp \left(\Lambda-\frac{e \Phi}{T_{e}}\right)+S_{n}
\end{aligned}
$$




$$
\begin{aligned}
\frac{\partial \nabla^{2} \Phi}{\partial t}= & \frac{c}{B}\left[\Phi, \nabla^{2} \Phi\right]+\frac{2 B_{0}}{c m_{i} R_{0}}\left(\frac{T_{e}}{n} \frac{\partial n}{\partial z}+\frac{\partial T_{e}}{\partial z}\right) \\
& +\nu \nabla^{4} \Phi+\frac{\sigma c_{s} m_{i} \Omega_{i}^{2}}{e R_{0}}\left[1-\exp \left(\Lambda-\frac{e \Phi}{T_{e}}\right)\right] \\
\frac{\partial T_{e}}{\partial t}= & \frac{c}{B}\left[\Phi, T_{e}\right]+\frac{4 c}{3 e R_{0} B_{0}}\left(\frac{7}{2} T_{e} \frac{\partial T_{e}}{\partial z}+\frac{T_{e}^{2}}{n} \frac{\partial n}{\partial z}-e T_{e} \frac{\partial \Phi}{\partial z}\right) \\
& +k_{e} \nabla^{2} T_{e}-\frac{2}{3} \frac{\sigma T_{e} c_{s}}{R_{0}}\left[1.71 \exp \left(\Lambda-\frac{e \Phi}{T_{e}}\right)-0.71\right] \\
& +S_{T} .
\end{aligned}
$$

Here, $S_{n}$ and $S_{T}$ are particle and heat sources, $\sigma=R / L_{c}=\Delta /\left(2 \pi L_{v}\right)$, and $[a, b]$ is the Poisson bracket. Experimental values are used for $R_{0} \sim 240 \rho_{s}, \Delta \sim 35 \rho_{s}$, $\sigma=0.056$, and $\Lambda=3$, with $\rho_{s}$ measured at the location of suprathermal ion injection. Diffusion coefficients are similar to experimental estimates, with $k_{e}=0.064 \mathrm{~m}^{2} / \mathrm{s}$ and $\nu=0.03 \mathrm{~m}^{2} / \mathrm{s}$. The sources are chosen to mimic the electroncyclotron and upper-hybrid resonance heating in TORPEX.

The code $^{15}$ used here to solve Eqs. (A2)-(A4) is based on a previously developed algorithm. ${ }^{47}$ Simulations are started with random noise and the constant plasma sources, producing a steepening of the gradient, which provokes the interchange instability and radial plasma transport balanced by losses to the walls. A quasi-steady state is achieved, and the data from this state are used as the input for the equation of motion for tracer particles.

${ }^{1}$ K. L. Wong, M. Ono, and G. A. Wurden, Rev. Sci. Instrum. 53, 409 (1982); E. D. Zimmerman and S. C. Luckhardt, J. Fusion Energy 12, 289 (1993); C. Riccardi, M. Fontanesi, A. Galassi, and E. Sindoni, Plasma Phys. Controlled Fusion 36, 1791 (1994); P. K. Sharma and D. Bora, ibid. 37, 1003 (1995); K. Rypdal and S. Ratynskaia, Phys. Rev. Lett. 94, 225002 (2005); K. W. Gentle and H. Huang, Plasma Sci. Technol. 10, 284 (2008).

${ }^{2}$ B. Li, B. N. Rogers, P. Ricci, and K. W. Gentle, Phys. Plasmas 16, 082510 (2009).

${ }^{3}$ A. Fasoli, B. Labit, M. McGrath, S. H. Mller, G. Plyushchev, M. Podestà, and F. M. Poli, Phys. Plasmas 13, 055902 (2006); A. Fasoli, A. Burckel, L. Federspiel, I. Furno, K. Gustafson, D. Iraji, B. Labit, J. Loizu, G. Plyushchev, P. Ricci, C. Theiler, A. Diallo, S. H. Mueller, M. Podestà, and F. Poli, Plasma Phys. Controlled Fusion 52, 124020 (2010).

${ }^{4}$ F. M. Poli, P. Ricci, A. Fasoli, and M. Podestà, Phys. Plasmas 15, 032104 (2008).

${ }^{5}$ P. Ricci and B. N. Rogers, Phys. Rev. Lett. 104, 145001 (2010).

${ }^{6}$ A. Bovet, A. Fasoli, I. Furno, K. Gustafson, and P. Ricci, "Investigation of fast ion transport in TORPEX," Nucl. Fusion (in press).

${ }^{7}$ G. Plyushchev, A. Diallo, A. Fasoli, I. Furno, B. Labit, S. H. Müller, M. Podestà, F. M. Poli, H. Boehmer, W. W. Heidbrink, and Y. Zhang, Rev. Sci. Instrum. 77, 10F503 (2006).

${ }^{8}$ K. Gustafson, P. Ricci, I. Furno, and A. Fasoli, Phys. Rev. Lett. 108, 035006 (2012); K. Gustafson and P. Ricci, Phys. Plasmas 19, 032304 (2012).

${ }^{9}$ W. Heidbrink and G. Sadler, Nucl. Fusion 34, 535 (1994).

${ }^{10}$ A. Fasoli, C. Gormenzano, H. L. Berk, B. Breizman, S. Briguglio, D. S. Darrow, N. Gorelenkov, W. W. Heidbrink, A. Jaun, S. V. Konovalov, R. Nazikian, J.-M. Noterdaeme, S. Sharapov, K. Shinohara, D. Testa, K. Tobita, Y. Todo, G. Vlad, and F. Zonca, Nucl. Fusion 47, 264 (2007).

${ }^{11}$ J. Jokipii, Astrophys. J. 146, 480 (1966).

${ }^{12}$ T. Hauff, F. Jenko, A. Shalchi, and R. Schlickeiser, Astrophys. J. 711, 997 (2010).

${ }^{13}$ M. A. Forman, R. Ramaty, and E. G. Zweibel, in Physics of the Sun, edited by P. A. Sturrock, T. E. Holzer, D. M. Mihalas, and R. K. Ulrich (D. Reidel Publishing Company, Dordrecht, 1986), Vol. 2, pp. 249-289.
${ }^{14}$ S. H. Müller, A. Fasoli, B. Labit, M. Mcgrath, O. Pisaturo, G. Plyushchev, M. Podestà, and F. M. Poli, Phys. Plasmas 12, 090906 (2005).

${ }^{15}$ P. Ricci and B. N. Rogers, Phys. Plasmas 16, 062303 (2009).

${ }^{16}$ S. Zhou, W. W. Heidbrink, H. Boehmer, R. McWilliams, T. Carter, S. Vincena, S. K. P. Tripathi, P. Popovich, B. Friedman, and F. Jenko, Phys. Plasmas 17, 092103 (2010).

${ }^{17}$ R. G. Kleva and J. F. Drake, Phys. Fluids 27, 1686 (1984); M. Vlad, F. Spineanu, J. H. Misguich, and R. Balescu, Phys. Rev. E 58, 7359 (1998); V. Naulin, A. Nielsen, and J. Rasmussen, Phys. Plasmas 6, 4575 (1999).

${ }^{18}$ S. V. Annibaldi, G. Manfredi, and R. O. Dendy, Phys. Plasmas 9, 791 (2002); K. Gustafson, D. Del-Castillo-Negrete, and W. Dorland, Phys. Plasmas 15, 2309 (2008); J. M. Dewhurst, B. Hnat, and R. O. Dendy, Plasma Phys. Controlled Fusion 52, 025004 (2010).

${ }^{19}$ M. Albergante, J. P. Graves, A. Fasoli, F. Jenko, and T. Dannert, Phys. Plasmas 16, 2301 (2009); T. Hauff, M. J. Pueschel, T. Dannert, and F. Jenko, Phys. Rev. Lett. 102, 75004 (2009). M. Albergante, J. P. Graves, A. Fasoli, M. Jucker, X. Lapillonne, and W. A. Cooper, Plasma Phys. Controlled Fusion 53, 054002 (2011).

${ }^{20}$ A. Zeiler, J. F. Drake, and B. Rogers, Phys. Plasmas 4, 2134 (1997).

${ }^{21}$ P. Ricci, C. Theiler, A. Fasoli, I. Furno, B. Labit, S. H. Müller, M. Podestà, and F. M. Poli, Phys. Plasmas 16, 055703 (2009).

${ }^{22}$ P. Ricci, C. Theiler, A. Fasoli, I. Furno, K. Gustafson, D. Iraji, and J. Loizu, Phys. Plasmas 18, 2109 (2011).

${ }^{23}$ I. Furno, B. Labit, M. Podestà, A. Fasoli, S. H. Mueller, F. M. Poli, P. Ricci, C. Theiler, S. Brunner, A. Diallo, and J. Graves, Phys. Rev. Lett. 100, 055004 (2008); I. Furno, B. Labit, A. Fasoli, F. M. Poli, P. Ricci, C. Theiler, S. Brunner, A. Diallo, J. P. Graves, M. Podestà, and S. H. Müller, Phys. Plasmas 15, 055903 (2008); M. Podestà, A. Fasoli, B. Labit, I. Furno, P. Ricci, F. M. Poli, A. Diallo, S. H. Müller, and C. Theiler, Phys. Rev. Lett. 101, 045001 (2008); C. Theiler, I. Furno, A. Fasoli, P. Ricci, B. Labit, and D. Iraji, Phys. Plasmas 18, 055901 (2011).

${ }^{24}$ G. Y. Antar, P. Devynck, X. Garbet, and S. C. Luckhardt, Phys. Plasmas 8, 1612 (2001); S. I. Krasheninnikov, D. A. D'Ippolito, and J. R. Myra, J. Plasma Phys. 74, 679 (2008); D. A. D’Ippolito, J. R. Myra and S. J. Zweben, Phys. Plasmas 18, 060501 (2011).

${ }^{25}$ T. A. Carter and J. E. Maggs, Phys. Plasmas 16, 012304 (2009); P. Popovich, M. V. Umansky, T. A. Carter, and B. Friedman, ibid. 17, 122312 (2010); B. N. Rogers and P. Ricci, Phys. Rev. Lett. 104, 225002 (2010).

${ }^{26}$ C. Birdsall and A. Langdon, Plasma Physics Via Computer Simulation (Taylor \& Francis, New York, 1991).

${ }^{27}$ T. M. Antonsen and B. Lane, Phys. Fluids 23, 1205 (1980).

${ }^{28}$ G. G. Howes, S. C. Cowley, W. Dorland, G. W. Hammett, E. Quataert, and A. A. Schekochihin, Astrophys. J. 651, 590 (2006).

${ }^{29}$ A. L. Newman and W. I. Newman, Phys. Fluids B 3, 915 (1991).

${ }^{30}$ B. D. G. Chandran, B. Li, B. N. Rogers, E. Quataert, and K. Germaschewski, Astrophys. J. 720, 503 (2010).

${ }^{31}$ W. Dorland, F. Jenko, M. Kotschenreuther, and B. N. Rogers, Phys. Rev. Lett. 85, 26 (2000); C. Estrada-Mila, J. Candy, and R. E. Waltz, Phys. Plasmas 12, 022305 (2005); Phys. Plasmas 13, 112303 (2006).

${ }^{32}$ A. B. Rechester and M. N. Rosenbluth, Phys. Rev. Lett. 40, 38 (1978); D. Del-Castillo-Negrete, Phys. Fluids 10, 576 (1998); P. Pommois, P. Veltri, and G. Zimbardo, Phys. Rev. E 59, 2244 (1999); D. Ruffolo, W. H. Matthaeus, and P. Chuychai, Astrophys. J. 597, L169 (2003); T. Hauff, F. Jenko, and S. Eule, Phys. Plasmas 14, 102316 (2007).

${ }^{33}$ R. Sánchez, D. E. Newman, J.-N. Leboeuf, V. K. Decyk, and B. A. Carreras, Phys. Rev. Lett. 101, 205002 (2008).

${ }^{34}$ H. J. Volk, G. Morfill, W. Alpers, and M. A. Lee, Astrophys. Space Sci. 26, 403 (1974).

${ }^{35}$ R. Huang, I. Chavez, K. Taute, B. Lukić, S. Jeney, M. G. Raizen, and E. L. Florin, Nature Phys. 7, 576 (2011).

${ }^{36}$ D. Del-Castillo-Negrete, B. A. Carreras, and V. E. Lynch, Phys. Rev. Lett. 94, 4 (2005).

${ }^{37}$ P. Ricci, B. N. Rogers, and S. Brunner, Phys. Rev. Lett. 100, 4 (2008).

${ }^{38}$ R. Sánchez, D. E. Newman, J.-N. Leboeuf, B. A. Carreras, and V. K. Decyk, Phys. Plasmas 16, 055905 (2009).

${ }^{39}$ S. Günter, G. Conway, S. da Graça, H.-U. Fahrbach, C. Forest, M. G. Muñoz, T. Hauff, J. Hobirk, V. Igochine, F. Jenko, K. Lackner, P. Lauber, P. McCarthy, M. Maraschek, P. Martin, E. Poli, K. Sassenberg, E. Strumberger, G. Tardini, E. Wolfrum, H. Zohm, and A. U. Team, Nucl. Fusion 47, 920 (2007).

${ }^{40}$ S. Zhou, W. W. Heidbrink, H. Boehmer, R. McWilliams, T. A. Carter, S. Vincena, and S. K. P. Tripathi, Phys. Plasmas 18, 082104 (2011). 
${ }^{41}$ S. Enge, G. Birkenmeier, P. Manz, M. Ramisch, and U. Stroth, Phys. Rev. Lett. 105, 175004 (2010).

${ }^{42}$ T. Hauff and F. Jenko, Phys. Plasmas 15, 2307 (2008).

${ }^{43}$ E. Fermi, Phys. Rev. 75, 1169 (1949).

${ }^{44}$ S. I. Braginskii, in Reviews of Plasma Physics (Consultants Bureau, New York, 1965), Vol. 1, p. 205.
${ }^{45}$ N. Bisai, A. Das, S. Deshpande, R. Jha, P. Kaw, A. Sen, and R. Singh, Phys. Plasmas 11, 4018 (2004); T. Rafiq, C. C. Hegna, J. D. Callen, and A. H. Kritz, ibid. 17, 022502 (2010).

${ }^{46}$ G. Q. Yu, S. I. Krasheninnikov, and P. N. Guzdar, Phys. Plasmas 13, 042508 (2006).

${ }^{47}$ V. Naulin and A. H. Nielsen, SIAM J. Sci. Comput. (USA) 25, 104 (2003). 